\title{
TRES ENFOQUES DE LA INVESTIGACIÓN SOBRE CONCEPCIONES ALTERNATIVAS
}

\author{
PINTÓ, R., ALIBERAS, J. y GÓMEZ, R. \\ Departament de Didàctica de la Matemàtica i de les Ciències Experimentals. \\ Universitat Autònoma de Barcelona.
}

\begin{abstract}
SUMMARY
In the last years the researchers on Science Education have given preferent attention to student's conceptions. Studies have been carried out and their interest is still growing. Our purpose in this paper is to give an approach to three ways of focusing the question: the «alternative conceptions», the «ways of reasoning» and the «causal mental models» approaches. Our goal is to compare them and show, without being exhaustive, some of their findings and future spectatives.
\end{abstract}

Las concepciones de los alumnos han sido objeto de atención preferente durante los últimos años para los investigadores en didáctica de las ciencias. La cantidad de estudios a que han dado Iugar es enorme y sigue creciendo (Duit, 1993). Junto a logros evidentes -cuestionar métodos đidácticos habituales, comprender mejor el proceso didáctico, generar nuevos modelos de enseñanza-aprendizaje, recibir aportaciones de la historia y de la filosofía de las ciencias...- se han registrado también decepciones (Gil, 1994). Nuestro propósito en este artículo es acercarnos a las distintas líneas de investigación, constatar sus resultados hasta ahora, y conjeturar posibles nuevos rumbos.

\section{LA INVESTIGACIÓN SOBRE CONCEPCIONES ALTERNATIVAS}

Hacia finales de la década de los setenta surge con fuerza una línea de indagación en el campo de la didáctica de las ciencias: se propone el estudio de las ideas científicas de los alumnos, especialmente en física, química y biología.

Estas ideas han recibido diversas denominaciones, cada una de las cuales transmite cierta concepción sobre su estatus (Driver y Easley, 1978; Duit, 1990): concepción alternativa, idea previa, estructura conceptual, error conceptual, ciencia de los alumnos, miniteorías...

Bajo esta perspectiva se investiga abundantemente durante algunos años, intentando detectar las ideas que los alumnos utilizan espontáneamente para enfrentarse a problemas o fenómenos científicos. Como resultado, disponemos hoy en día de extensos catálogos de tales ideas, referidas a los más variados campos científicos, así como de algunos de los modelos conceptuales que parecen utilizar. Pueden encontrarse recopilaciones de tales resultados en muchas fuentes; en lengua castellana, por ejemplo, Hierrezuelo y Montero (1988) y Driver, Guesne y Tiberghien (1989).

Además de detectar ideas alternativas, ciertas investigaciones se han propuesto caracterizar sus propiedades, para así poder fundamentar mejor las estrategias didácticas. Algunas de dichas posibles propiedades serán analizadas a continuación: coherencia, universalidad, persistencia y consistencia.

\section{Coherencia}

Aunque no hay unanimidad en la terminología, aquí llamaremos coherente una concepción si no presenta contradicciones internas. 
Algunas de Ias diversas denominaciones que han recibido las concepciones de los alumnos muestran el grado de coherencia que el autor les supone. Así, los términos «conceptual framework» (oestructura conceptual), «teoria», «ciencia», etc., ya sugieren algo estructurado, dan idea de interconexión entre conceptos, aunque sea con un grado de coherencia limitado.

Clement (1983) afirmaba que se trata de un sistema de concepciones interconectadas coherente que resulta suficientemente estable, lo que explicaria su resistencia al cambio. Tales sistemas conceptuales en algunos casos presentan semejanzas con teorías propuestas a lo largo de la historia de la ciencia hoy ya abandonadas: sería el caso, por ejemplo, de las concepciones sobre furerza y movimiento, respecto de la teoría medieval del «ímpetus».

Para Driver(1981), el conocimiento científico del alumno está interconectado, aunque no siempre de forma explícita o consciente. Cierta coherencia se detecta en algunas concepciones de los alumnos, correspondientes a modelos teóricos distintos de los vigentes en la ciencia actual. Otras concepciones, aun sin corresponder a tales modelos teóricos parecen tener una lógica interna. Finalmente otras concepciones, que parecen provenir de una asimilación inadecuada dela instrucción recibida, muestran un grado variable de coherencia.

Si algunas concepciones alternativas muestran una coherencia interna y, en cambio, no se observa en otras, podría argumentarse que la necesidad de coherencia sólo es propia del discurso científico y que, por lo tanto, los alumnos pueden carecer de tal experiencia personal. Pero este argumento no parece suficiente: si así fuera, por ejemplo, no se incomodarían cuando se les enfrenta con lo que consideramos contradicciones internas de su discurso.

Acerca de la coherencia interna o la incoherencia de las concepciones alternativas sigue, pues, abierta una polémica sobre la que se está investigando.

\section{Universalidad}

¿Son universales las concepciones alternativas?; es decir, ¿inuestran concepciones parecidas los estudiantes đe diferentes países o culturas? Cierto número de investigaciones se han encaminado a resolver esta cuestión, como Driver (1985) y Shipstone y otros (1988). Las referidas a concepciones de los alumnos sobre circuitos eléctricos, realizadas en varios países europeos, parecen apuntar a ideas semejantes para comunidades distintas: intensidad decreciente a lo largo de un circuito, distribución del voltaje en las derivaciones, influencia de la forma de presentación del circuito, etc. (Fig. 1). Se aprecia similitud en la frecuencia de aparición de las diversas concepciones sobre electricidad básica en cada país. Las pequeñas diferencias que se pueden observar son fácilmente atribuibles a la diferente organización de los respectivos currículos.
Figura I

Ejemplo de investigación siguiendo las líneas de exploración de concepciones alternativas.

En el circuito siguiente, todas las bombillas son del mismo tipo. Completa las intensidades $I_{1}, I_{2}$ e $I_{3}$ en los cables.

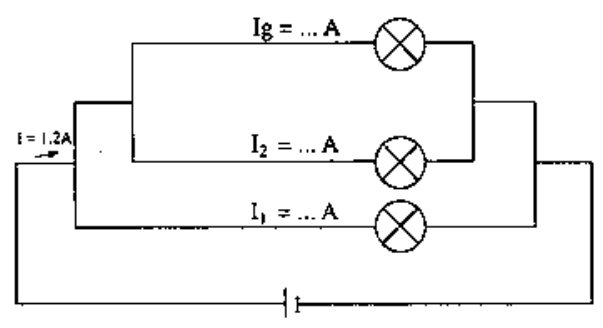

Porcentajes de cada tipo de respuesta en cinco países europeos, segúa Shipstone y otros (1988). Puede verse el efecto de la forma de presentación del circuito: dibujando las derivaciones en forma simétrica, los aciertos aumentan.

\begin{tabular}{|c|c|c|}
\hline País & $\begin{array}{c}\text { Respuesta } \\
0.4 / 0.4 / 0.4 \\
\text { correcta }\end{array}$ & $\begin{array}{c}\text { Respuesta } \\
0.6 / 0.3 / 0.3\end{array}$ \\
\hline Suecia & 14 & 59 \\
\hline Francia & 21 & 60 \\
\hline Holanda & 17 & 57 \\
\hline Baden-Württenberg & 18 & 53 \\
\hline Hesse & 12 & 31 \\
\hline Inglaterta & 15 & 49 \\
\hline
\end{tabular}

Sin embargo, no faltan estudios en los que los estudiantes manifiestan ideas distintas en contextos culturales distintos. Por ejemplo, Hewson y Hamlyn (1984), al investigar las ideas sobre el calor que mostraban adolescentes de lengua y cultura africanas, encontraron concepciones más cercanas a las científicas que las que aparecen habitualmente en los países occidentales. El análisis de este resultado parece indicar que los factores culturales, ambientales y lingiísticos no son ajenos a la construcción de las ideas alternativas.

Otro factor del que podría esperarse cierta influencia es Ia propia constitución y funcionamiento del aparato cognitivo humano. Dado que tanto sus posibilidades como sus limitaciones deben ser generales, parece lógico esperar que contribuya en cierta medida a la universalidad en las concepciones. Efectivamente, el trabajo de Mali y Howe (1979) sobre concepciones de los alumnos nepalfes respecto a la Tierra en el espacio, reproduciendo uno anterior de Nussbaum y Novak (1976) entre alumnos occidentales, encontró la misma secuencia de concepciones.

En definitiva, estos estudios y otros posteriores parecen demostrar cierto grado de universalidad en las concepciones, si bien no debe olvidarse la intervención de factores relacionados con el contexto social y natural. 


\section{Persistencia}

Uno de los aspectos más preocupantes de las concepciones de Ios alumnos es la constatada estabilidad de tales ideas, su importante resistencia al cambio. Dichas concepciones persisten a lo largo de períodos muy dilatados de tiempo, y ello a pesar, incluso, de intervenciones educativas dirigidas a facilitar su transformación (Driver y Erickson, 1983). Se ha comprobado que las concepciones de base experiencial són más persistentes que otras (Engel y Driver, 1986).

Dupin y Joshua (1987), al analizar el pensamiento de los alumnos sobre los circuitos eléctricos, diferencian dos tipos de concepciones alternativas: unas superables mediante la enseñanza y otras más resistentes al cambio. Éstas, detectables en alumnos de distintos países, serían más universales y fruto de esfuerzos cognitivos parecidos para enfrentarse con los problemas y con el aprendizaje.

Por todo ello, la comprobada persistencia de muchas concepciones obliga a moderar ciertas expectativas respecto a los programas didácticos: no podremos esperar que determinados aprendizajes se realicen en unas pocas horas. Sustituir concepciones generadas a to largo de toda la vida requiere su tiempo (Driver, 1986). Todo ello nos obliga a planifícar cada aprenđizaje importante más en términos de años que de horas.

\section{Consistencia}

Diremos que un alumno es consistente en la utilización de una concepción determinada cuando la utiliza en contextos distintos, aunque científicamente equivalentes. Así diferenciamos consistencia de coherencia, entendida esta última como concepciones con una lógica interna. La coherencia corresponde al discurso, la consistencia al alumno.

El estudio de la consistencia con que las personas utilizan sus concepciones alternativas tiene especial importancia para la didáctica. En efecto: si se consiguiese probar la consistencia en la utilización de las ideas alternativas, podría asignarse a cada alumno una categoría conceptual que caracterizaría sus explicaciones. Como consecuencia, a partir de este diagnóstico claro de la situación de partida, podría diseñarse ađecuadamente una estrategia de cambio específica. Si no se diera consistencia, no podría contarse con tal recurso.

Los primeros artículos que abordaban esta cuestión (Caramazza, 1981; Clement, 1983) exponían su creencia en la utilitzación consistente de las ideas alternativas por parte de los alumnos, dado su carácter fuertemente estructurado.

En cambio, investigaciones posteriores se han encargado de desmentirlas. Engel y Driver (1986) encontraron consistencia aparente a nivel de grupo, consistencia que desaparecía al analizar los resultados individualmente. Tampoco encontraron consistencia en grado significati- vo Licht y Thijs (1990), Finegold y Gorsky (1991), Pintó (1991) ni Millar (1993).

La polémica parece haber dejado claro que los estudiantes no muestran, en general, demasiada consistencia en su utilización de concepciones alternativas. Sin embargo, parecen mostrar mayor consistencia los alumnos mayores cuando tienen que aplicar ideas científicas.

Las concepciones alternativas parecen utilizarse siguiendo reglas específicas para situaciones específicas, en vez de aplicar leyes generales. El grado de consistencia depende del contexto. Por ejemplo, el dominio de un tema científico desde el punto de vista teórico no implica su utilización correcta ante una situación práctica. El lenguaje mismo utilizado en la pregunta o ciertos aspectos del contexto en que se sitúa el problema pueden infuir, asimismo, en la utilización de una concepción u otra. Se han obtenido resultados distintos planteando la misma situación en un contexto cotidiano o en un contexto «científico», por ejemplo, al intentar explicar el enfriamiento de agua caliente en un vaso de precipitados, o el papel del jersey en invierno (Equip ICE, UAB, 1993). Formular el principio de conservación de la energía o la segunda ley de la termodinámica es algo trivial para los alumnos con ciertos niveles de instrucción, pero muy pocos de los mismos alumnos son capaces de dar correcta cuenta del significado, por ejemplo, de la idea de «crisis energética mundial» (Pintó, 1991). Parece evidente pues, que los alumnos no siempre categorizan de la misma manera aquellas situaciones que para los científicos son similares.

Las diferentes características del pensamiento científico y el pensamiento cotidiano analizadas por Reify Larkin (1991) y Pozo (1992) pueden dar cuenta de la disparidad de las respuestas de los alumnos ante cuestiones científicamente iguales. Solomon apuntaba ya en 1982 cuán importante es ayudar y enseñar a establecer el puente entre dominio cotidiano y dominio científico.

Así pues, la falta de consistencia dificulta la tarea del profesor: no se encuentra ante unos cuerpos teóricos sólidos -aunque sean científicamente erróneos- utilizados consecuentemente, sino ante diversas concepciones que se activan por criterios distintos a los científicos.

\section{Implicaciones didácticas: cambiar de concepción}

La existencia y el poder de las ideas alternativas hace imposible sostener una concepción de la enseñanza como simple transmisión de información: los aprendizajes científicos importantes han de consistir más en cambiar unas formas de ver las cosas-pasar de las concepciones alternativas a las concepciones científicas- que en acumular unas informaciones. Se trata del Ilamado cambio conceptual.

EI modelo didáctico más conocido de cambio conceptual es el de Posner y otros (1982), basado en modelos filosoficos del progreso científico, especialmente el de Toulmin y el de Kuhn. 
Para explicar la evolución de los conceptos, teorías y métodos en las ciencias, Toulmin (1977) tomó como analogía la teoría de Darwin sobre la evolución de las especies. Los mecanismos de innovación y selección serían parecidos en ambos casos. Según esta analogía, la aparición de nuevos conceptos en una ciencia no es suficiente para que se impongan a los anteriores; para que lleguen a incorporarse tendrán que demostrar su superioridad de acuerdo con los métodos aceptados por los científicos y con las finalidades que persiguen (Aliberas, Gutiérrez e Izquierdo, 1989).

Posner y otros (1982) realizan todavía orra analogía sobre la anterior: la de comparar el aprendizaje de las ciencias con el cambio científico. Aprender ciencias sería un proceso de innovación y selección (conceptual) parecido al que realizan los científicos en su trabajo, y parecido también al caso (biológico) darwiniano. Según ello, adquirir nuevos conceptos no sería suficiente; tendrán que imponerse a los conceptos anteriores y desplazarlos. Aun sin dejar de ser racionales, los mecanismos de selección no son de naturaleza lógica (a pesar de la presentación que suele aparecer en los libros de texto): su finalidad no es descubrir «la verdad» sino resolver pragmáticamente las dificultades.

Hay que notar que en el artículo del año 1982 los autores hablan cle cambio conceptual comparándolo también con la «revolución científica» de Kuhn: un proceso de cambio, que puede acabar en éxito o en fracaso, pero relativamente rápido y con resultado claro.

Durante la década de los ochenta se han realizado muchos esfuerzos para diseñar estrategias didácticas de enseñanza, encaminadas principalmente a conseguir el cambio conceptual. Desgraciadamente, no conocemos ningún estudio sistemático de sus resultados. Los que se han publicado son desiguales: aunque todos parecen positivos, el grado de efectividad es muy variado. En general, los resultados no son espectaculares. Sin ser decepcionantes, no llegan a ser suficientemente positivos. Ha quedado la sensación de que posiblemente hay algo que se escapa.

Años después del primer artículo, uno de los autores del modelo de cambio conceptual (Hewson y Thorley, 1989) parece rectificar su idea sobre la cuestion: ya no sería un cambio revolucionario, sino que más bien consistiría en un debilitamineto del estatus de las concepciones previas (en lo referente a insatisfacción, inteligibilidad, plausibilidad y utilidad) y en un fortalecimiento simultáneo del estatus de las nuevas.

La doble analogía sobre la que descansa el modelo de Posner y otros (1982) también debe estudiarse atentamente, ya que en todas las analogías hay semejanzas, pero también diferencias. Por un lado, los modelos evolutivos de ciencia como el de Toumin han sido criticados por filosofos cognitivos como Thagard (1988): ni la variación de concepción se produce de manera ciega, ni la selección se hace por criterios locales, ni la transmisión del conocimiento es genética. Incluso tales críticas consideran que la metáfora darwiniana ayuda a esconder aspectos cruciales del trabajo científico, como el problema de la inducción.

Por otro lado, es evidente que entre el descubrimiento científico y el aprendizaje de las ciencias hay diferencias profundas (Reif y Larkin, 1991): diferentes metas, diferentes formas de razonamiento, diferente estructura del conocimiento, diferentes métodos...

Una de las consecuencias es la necesidad, cada vez más clara, de no reducir el cambio en el aprendizaje al nivel conceptual. Gil (1986) propone un cambio conceptual y metodológico: no sólo hay que cambiar lo que se piensa, sino también la manera cómo se enseña. En la misma línea, Hewson (1985) considera que los compromisos epistemológicos (especialmente la necesidad de consistencia interna y de generalizabilidad) también pueden ser objeto de cambio conceptual. Según Hashweh (1986), el cambio conceptual sólo se puede dar cuando la epistemología cientffica sustituya Ia anterior; es decir; cuando el requerimiento de coherencia entre las concepciones -y entre éstas y las evidencias empíricas-, el acento en Ia objetividad, la exigencia de formulación sin ambigüedades de teorías empíricamente refutables, etc. constituyan una metodología sistemática para validar el conocimiento.

Finalmente, West y Pines (1983) aún van más lejos al hacer notar que en el proceso de cambio conceptual están implicados factores que no son racionales, como los estéticos y los afectivos.

A la vista de todo ello, nos parece evidente la necesidad de introducir modificaciones en nuestras maneras de conceptualizar el aprendizaje en ciencias y de enfrentarnos a su enseñanza. El cambio conceptual sólo se dará a lo largo de un proceso gradual cuando el alumno vaya requiriendo una coherencia entre sus concepciones y las evidencias empíricas, cuando la objetividad sea un deseo fuertemente sentido; en definitiva, cuando vaya adquiriendo una metodología para validar el conocimiento y, a la vez, las nuevas concepciones no le aparezcan como refutables por motivos personales. Nuevas metodologías de enseñanza son, pues, necesarias, aunque conviene tener presente que van a producirse progresos y regresiones.

\section{LA INVESTIGACIÓN SOBRE FORMAS DE RAZONAMIENTO}

Si bien muchos investigadores han orientado sus esfuerzos en describir minuciosamente las concepciones alternativas de los alumnos, otros se han propuesto el análisis de las explicaciones de los alumnos buscando cierta estructura en ellas, tratando de encontrar alguna visión científica propia de los alumnos que las justifique (Viennot, 1973).

En tal dirección han ido diversas investigaciones en los últimos años (Sanmartí, 1989), ya que detectar múltiples 
concepciones alternativas y disponer sólo de una amplia descripción no resulta suficiente para colmar el deseo común de interpretaciones más profundas. A medida que se ha ido avanzando en el conocimiento de las concepciones alternativas, ha resultado más necesario explicarlas en términos más fundamentales. El propósito es parecido al que llevó a dar cuenta de la gran diversidad de substancias mediante un número limitado de elementos químicos.

En esta línea, se ha intentado inferir cual podía ser la lógica interna de las argumentaciones de los alumnos, dando por supuesto que tales argumentaciones podfan ser distintas de las utilizadas en la ciencia. En un primer momento se trataba de encontrar estructuras lógicas generales en la diversidad de respuestas de cada alumno sobre un mismo problema científico presentado desde perpectivas distintas. Sin embargo, diversos estudios apuntan también a la falta de consistencia de los alumnos en sus razonamientos (Finegold y Gorsky, 1991; Pintó, 1991). Algunos argumentan que la diversidad de razonamientos de un mismo alumno ante problemas similares puede explicarse por el hecho de que los alumnos se rigen sólo por criterios pragmáticos. «Los criterios de utilidad dominan sobre los de generalizabilidad o la economía de esfuerzon (Driver 1993). Si así fuera, sólo podríamos esperar inconsistencia.

De todos modos, es difícil aceptar que las distintas argumentaciones đe los alumnos no tienen lógica alguna (cierto hilo conductor, denominador común, patrón o esquema interno), a pesar de que puedan utilizar razonamientos diferentes según la situación a la que se enfrenten.

En esta búsqueda de unos patrones de razonamiento que procedan de alguna forma de elaborar la información está, en nuestra opinión, el mayor interés de las aportaciones de la que podemos denominar corriente deI LDPES de París.

Como dice Viennot (1988), «el estudio de campos conceptuales asociados a dominios específicos de la física (mecánica, electrocinética, propagación de ondas, termodinámica, etc.) ha hecho aparecer unas tendencias de razonamiento que, evidentemente, escapan de los Í́mites de estos campos específicos y se manifiestan bajo formas análogas de un dominio a otro».

La existencia de unos modos o patrones de razonamiento podría dar pautas para comprender la resistencia de las concepciones alternativas. Los verdaderos obstáculos para cualquier cambio conceptual serían ciertos modos de razonamiento bien anclados.

Ya Bachelard en 1938 se refería al animismo, al antropomorfismo, a la necesidad de substancialización de los conceptos, al obstáculo verbal, etc. como obstáculos epistemológicos para la producción científica. Siguiendo la tradición de Bachelard, los obstáculos epistemológicos pueden dar cuenta de diversos tipos de concepciones alternativas que se han encontrado en dominios conceptuales bien distintos. Encontra- mos buena parte de tales obstáculos en las explicaciones de los alumnos.

Es este carácter transversal de los obstáculos epistemológicos, lo que nos permite superar el "catălogo de concepciones alternativas» para cada campo conceptual. Al observarlas desde una perspectiva más general, se tiene una mejor visión de las raíces profundas deI problema y, por tanto, se pueden comprender mejor las dificultades que aparecen al pretender superarlas.

Nos detendremos a continuación en un par de tales modos de razonamiento.

\section{Reducción funcional}

Viennot (1988) describe la reducción funcional como la tendencia a no tener presentes todas las variables que intervienen en un problema concreto.

Ya InheIder y Piaget (1955) señalaban la tendencia a razonar con una sola variable a la vez, pero la reducción funcional, como la concibe Viennot, tiene también otras características. Por ejemplo, en los sistemas en que se afirma la constancia de ciertas magnitudes, el alumno tiende a no tener presentes las variables de las que el valor de la constante es independiente. Así, en un circuito eléctrico en el que el genenerador tiene una fem constante, según la ley de $\mathrm{Ohm}$, al disminuir la resistencia, aumenta la intensidad y viceversa. Pero, ¿qué ocurre si aumenta la temperatura? Pocos alumnos se dan cuenta que $\mathrm{R}$ aumenta con la temperatura, con lo cual también disminuirá I.

Otras veces, mediante el razonamiento funcional, el alumno tiende a no considerar que el valor que tengan ciertas variables puede hacer variar el valor de la constante. Así, señala Viennot, se afirma que la velocidad de la luz es constante sin tener en cuenta que el valor de esta constante depende del medio en que se propaga. El medio constituye una variable olvidada.

Tal reducción funcional implica también no diferenciar nociones relacionadas. Así se manipulan como si se tratara de un mismo concepto: velocidad y aceleración, o bien temperatura y calor, o energía y trabajo, o intensidad y voltaje, o movimiento-fuerza-impulso-energía, etc. En todos estos casos, diversas variables son consideradas prácticamente una sola, con to que el número efectivo de variables utilizadas es inferior al adecuado.

Otra de Ias modalidades de reducción funcional es la utilización de razonamientos causales lineales.

\section{Razonamientos lineales causales}

Al considerar problemas de varias variables, a menudo nos encontramos con argumentos construidos como cadenas lineales en donde cada fenómeno se especifica con una sola variable, y los nexos entre elios son del tipo: «una causa $\rightarrow$ un efecto», y ello a pesar de que otras 
causas puedan desempeñar un papel importante. Estas otras causas son obviadas, y de ahí que también se considere a este caso una forma de reducción funcional.

Parece como si el alumno considerara que sólo una causa es necesaria para conseguir cierto efecto. Así, por ejemplo, para analizar una compresión adiabática de un gas, se hace la siguiente cadena:

Si el volumen disminuye (se supone constante el número de moles) > el número de choques por unidad de superficie y de tiempo sobre las paredes aumenta $>$ la presión aumenta.

Es un encadenamiento insuficiente para dar cuenta deI fenómeno. Hay que añadir al primer efecto, un efecto de aumento de la velocidad media de las partículas ya que participa en el aumento de la presión. La variable velocidad (cuadrática media) se olvida como causa del aumento de presión. Se trata pues de una reducción.

No se suele tener en cuenta que cada causa puede dar lugar a diversos efectos, ni que cada efecto no tiene por qué ser el resultado de una sola causa.

Las flechas del razonamiento lineal anterior son, aparentemente, conexiones causales. Pero un análisis más atento permite darnos cuenta đe que contienen también una significación temporal. Dicha cadena suele leerse: «Si el volumen disminuye, entonces el número de choques aumenta...", donde la palabra «entonces» tiene un doble significado, conclusivo («por lo tanto») y temporal («a continuación»). Al inducirse una cronología se entra en contradicción con la teoría de los fenómenos cuasiestáticos según la cual el valor de las diferentes magnitudes varía simultáneamente.

Por otro lado, la đescripción estấ centrada en la evolución a lo largo del tiempo de un solo objeto: el gas perfecto, sin tener en cuenta la interacción gas-pistón, lo cual podría conducir a no darse cuenta del aumento de la velocidad de las partículas.

Tal tipo de razonamiento se ha detectado en campos tan diversos como termodinámica (Rozier, 1988), electrodinámica (Closset, 1983), mecánica (Fauçonnet, 1983)o propagación de señales mecánicas (Maurines, 1986).

Debido al carácter de secuencia temporal de los argumentos, resulta más fácil para los alumnos explicar situaciones de cambio (por qué cae un objeto, por ejemplo) que estados de equilibrio (como un objeto que se mantiene sobre el suelo): al no observarse ningún efecto, no ven ninguna necesidad de buscar una causa, por lo que suelen manifestar que no encuentran nada que explicar.

En algunos casos, Ia disposición espacial favorece la utilización del razonamiento causal lineal. Es el caso de los circuitos eléctricos: típicamente son analizados por
Ios alumnos secuencialmente, sin tener en cuenta que forman un sistema. Por ejemplo, al estudiar el comportamiento de un componente no consideran los demás elementos que «vienen después» (Fig. 2).

Como puede verse, mediante un mismo modo de razonamiento pueden explicarse concepciones alternativas de campos conceptuales bien diversos.

\section{Figura 2}

El mismo ejemplo, interpretado ahora siguiendo las líneas de investigación sobre formas de razonamiento

Los resultados incorrectos de la figura 1 pueden explicarse por un razonamiento causal Inneal: al llegar la corriente a la bifurcación "entonces", al encontrar dos caminos, la corriente se divide en dos mitades (sin hacer caso de lo que pueda "venir después"). I.os estudiantes encontraron muy fácil la pregunta. Al ignorar factores que intervicner en el fenomeno, se ha producido una "reduccion funcionat".

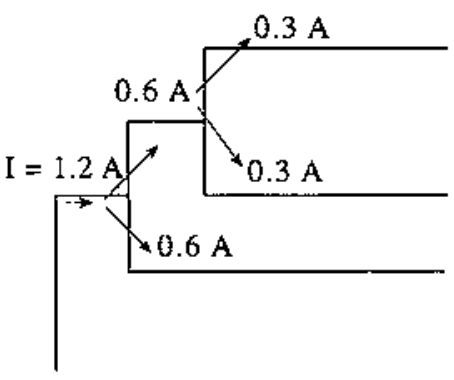

\section{Implicaciones didácticas: cambiar las formas de razonar}

Si buscar formas de razonamiento resulta más fructífero que describir concepciones particulares es debido a la generalidad de las formas de razonamiento: no se circunscriben a un dominio conceptual concreto, sino que persiguen la descripción global de las formas de razonar que operan en los distintos ámbitos. Además, también permiten abordar con más profundidad las causas de la resistencia al cambio.

Dicen Joshua y Dupin (1993) que «las capacidades de adaptación de ciertas concepciones y modos de razonamiento de los alumnos, su pertinencia para un tipo de problemas, la coexistencia entre concepciones y su reagrupamiento en registros estancos, así como la existencia de razonamientos transversales no pertinentes, explican en gran medida la persistencia de estas concepciones y razonamientos, a la vez que indican que su superación sólo puede ser el resultado de un trabajo didáctico de gran alcance.»

Dichos autores (1993) proponen un mismo tratamiento para la superación de las concepciones alternativas y de los modos de razonamiento. Consideran que la resistencia al cambio conceptual de las concepciones alternativas y de los modos de razonamiento puede explicarse por la gran plasticidad de las concepciones de los estudiantes para dar respuesta a situaciones muy diversas. Esta posibilidad de utilizar unas concepciones alternati- 
vas u otras ante cuestiones que los estudiantes consideran distintas - aunque puedan ser equivalentes desde un punto de vista científico- les daría menor vulnerabilidad. No es necesario romperlas porque ya son muy adaptables, flexibles; y pueden adaptarse a la resolución de numerosos problemas.

Por su parte, Astolfi (1993) se pregunta sobre si conviene abordar la superación de los obstáculos a partir de sus manifestaciones en casos concretos, o bien si es más eficaz atacarlos de forma transversal, global. «Si se tratan estos obstáculos aisladamente, se corre el riesgo de ocuparse más de sus manifestaciones contingentes que de su raíz, y habrá que reproducir el proceso para cada ejemplo, sin que los alumnos vean necesariamente que se trata del mismo obstáculo. Pero si por el contrario se trabaja sobre ellos más globalmente, hay que asegurarse que se produce una transferencia efectiva de este aprendizaje general a cada situación particular... La respuesta sólo puede ser dialéctica, es decir, difícil, jugando con la alternancia de situaciones, en las que un mismo obstáculo será, alternativamente, trabajado global y particularmente.»

Rozier (1988) insiste especialmente en dos recomendaciones para que los razonamientos de los alumnos no sean razonamientos lineales causales:

- Fomentar el análisis de sistemas y no de objetos. Así se puede favorecer que los razonamientos no estén centrados en objetos sino en las interacciones entre sistemas.

- Insistir en la simultaneidad. No caer en la tentación, en aras de una más facil comprensión, de explicar historias para dar cuenta de un fenómeno. La tendencia a inventar secuencias cronológicas es un recurso frecuente en muchos ámbitos de la vida cotidiana. Dice Rozier: «Conviene que los profesores tengan la maestría de administrar este delicado equilibrio entre el entusiasmo que provoca una entrada en resonancia con el razonamiento lineal causal y los áridos caminos de la teoría física.»

\section{¿INVESTIGACIÓN SOBRE MODELOS MENTALES?}

La reciente utilización en la investigación sobre diđáctica de las ciencias del concepto de modelo mental puede significar el inicio de una nueva e interesante línea en los estudios sobre las ideas científicas de los alumnos, de la cual podrian derivarse importantes consecuencias educativas. Resnick (1989) afirma que, en el lenguaje de Ia ciencia cognitiva, aprender acerca de algo, llegar a comprenderlo, es construir un modelo mental. Si bien, como dice Rogers (1992), «los modelos mentales están siendo utilizados actualmente para explicar un amplio rango de fenómenos psicológicos», nosotros vamos a centrarnos en como pueden contribuir a la investigación en didáctica de las ciencias.

\section{Modelo mental}

Entendemos por modelo mental el constructo psicológico que se forman los individuos al interaccionar con otras personas, con el medio o con algún artefacto tecnológico, y que les permite dar cuenta de tal interacción y predecir el comportamiento de los sistemas en futuras relaciones (Johnson-Laird, 1983; Gentner y Stevens, 1983).

Construimos modelos mentales para ayudarnos a comprender el funcionamiento de un sistema físico o social en circunstancias muy diversas. Por ejemplo, para abrir una puerta, utilitzar un teléfono, un abrelatas o para accionar un dispositivo, de los que desconocemos el funcionamiento, construimos modelos mentales de cómo funcionan. Y si el sistema se nos resiste, solemos atribuir el fracaso a causas que no habíamos previsto, lo cual nos permite construir nuevos modelos para guiar nuevos intentos (Norman, 1990).

\section{Construir un modelo mental requiere:}

- Hacerse una representación interna del sistema. Es decir, «traducirnos» Ios elementos de la realidad a un código propio en función de nuestros intereses.

- Utilizar un proceso de inferencia (que no tiene por qué ser un proceso de lógica deductiva).

- Poner en marcha en nuestra mente un proceso de simulación cualitativa del funcionamiento del sistema exterior que estamos analizando; es lo que se denomina «ejecutar» el modelo. Esto permite comparar los resultados de la ejecución con lo que está sucediendo en el sistema real. Por lo tanto, permite al sujeto evaluar su modelo mental y, si es necesario, corregirlo.

Utilizar el concepto de modelo mental para estudiar el razonamiento humano es una alternativa interesante cuando la lógica deductiva no parece ser la utilizada por los individuos.

Adoptar tal punto de vista puede ser una forma de conseguir comprender este cúmulo de razonamientos y concepciones alternativas de los alumnos que no acertamos a explicarnos.

\section{Utilidad del «modelo mental mecánico» de De Kleer y Brown}

Utilizando el «modelo mental mecánico» de De Kleer y Brown(1983), Gutiérrez (1994) ha conseguido dar cuenta no sólo de las ideas que los alumnos aplican en situaciones cotidianas relacionadas con fuerzas y movimientos, sino también de cómo se realiza su evolución.

Dicho modelo - procedente del campo de la inteligencia artificial- es demasiado complejo para ser resumido aquí de manera comprensible. Se trata de un modelo teórico formalizado muy estrictamente en su forma ori- 
ginal. Por ello, vamos a exponer sólo algunas de sus características y de sus logros al aplicarlo a la didáctica de las ciencias. Pretendemos así mostrar lo prometedor de esta vía.

Para situar brevemente el modelo de De Kleer y Brown, diremos que proviene de un ámbito de la inteligencia artificial que se propone la construcción de máquinas dotadas de «sentido común» en el terreno de la física, con la finalidad de «comprender» el cambio físico. Entre las distintas líneas que coexisten en este terreno, dichos autores han optado per una modelización cualitativa de los fenónenos, así como por un sistema de inferencia basado en la causalidad.

Llevando este modelo al terreno didáctico, un alumno se enfrentaría a una situación científica concreta construyendo un modelo mental cualitativo de esa situación. Pero la diferencia clave con otras líneas de investigación sobre modelos mentales consiste en que, según dicho enfoque, el individuo no realizaría inferencias a base de procesos lógicos o legales, sino causales. Por ejemplo, hay alumnos que no utilizan el principio de inercia como una ley que se aplica cuando se dan las condiciones para ello (pensamiento legal), sino que piensan que todo efecto debe tener una causa; entonces, asumiendo que el movimiento es un efecto, se les hace necesaria una causa (pensamiento causal), que suelen identificar con alguna fuerza. Todo ello les dificulta notablemente la comprensión del principio de inercia.
El alumno hace evolucionar sus modelos mentales causales (Gutiérrez y Ogborn, 1992; Gutiérrez, 1994) persiguiendo mejorar la satisfacción de tres restricciones:

- no tener contradicciones internas (coherencia),

- tiene que dar cuenta de la realidad (correspondencia), - debe resultar útil en situaciones nuevas (robustez).

El modelo teórico citado ha permitido (Gutiérrez y Ogborn, 1992), por ejemplo:

- Describir - a partir de una nueva técnica de entrevista clínica llamada «teachback»- el modelo mental que un alumno utiliza en un momento dado para analizar una situación científica concreta, așí como comprender los cambios que progresivamente introduce en dicho modelo. (La entrevista «teachback» consiste en un tipo de conversación en la que entrevistador y entrevistado llegan a un consenso sobre el pensamiento de éste úlitimo).

- Reanalizar, en términos de modelos mentales (y razonamiento causal) los datos obtenidos en otras investigaciones; datos que habían sido interpretados a base de variables (y razonamiento lógico) o de modos de razonamiento.

La utilización đe la entrevista «teachback» ha permitido a Gutiérrez (1994) descubrir -a pesar de los resultados

Si pudiérarnos conversar con uno de los alumnos que respondió incorrectamente a la situación planteada en la figura 1 para conocer el mođelo mental que há utilizado, probablemente nos hablaría de algún tipo de material que circula por los cables.

Procedente de la pila, a ese material no le sucedería nada digno de mención hasta que llegara a la primera bifurcación. Principios tales como «la catusa debe estar estructuraimente próxima a su efecto» y «no hay causalidad estructural» explican que el alumno sólo se fije en la bifurcación.

Alli se partirfa en dos mitades sirnétricas, a causa de la simetría de la bifurcación misma (la misma causa tiene que producir el mismo efecto). Probablemente el alumno negaría que la parte restante del circuito pueda infiłuir en lo quc en este punto ocurre. El principio de que alas causas preceden a sus efectosn le conduce a imaginar un episodio para cada bifurcación. Estos no serían simultáneos sino sucesivos.

Para hacer evolucionar su modelo mental podemos intentar diversas vías; por ejemplo, hacerie comprobar que las lecturas de los amperimetros en distintos puntos del circuito son distintas de las que había previsto (falta de correspondencia). Ello le obligaráa introducir cambios en su modelo mental del circuito, con la finalidad de dar cuenta de lo que en realidad ocurre (búsqueda de correspondencia).

Otra posibilidad sería hacerle comparar el circuito propuesto con el que sigue:

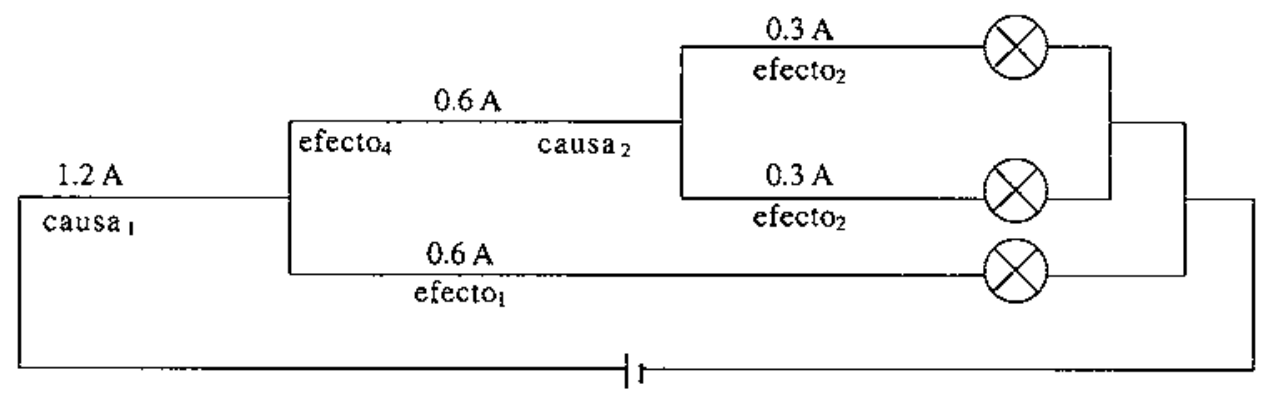


Si el alumno se da cuenta de que ambos circuitos son equivalentes, puede llegar a notar que su modelo conduce a resultados contradictorios (falta de coherencia) o que no es aplicable en todas las condiciones en que deberia (falta de robustez). No puede seguir aplicando el constructo según el cual el material que circula se subdivide en partes iguales siguiendo las simetrfas que observa. Sentirá la necesidad de modificar su modelo.

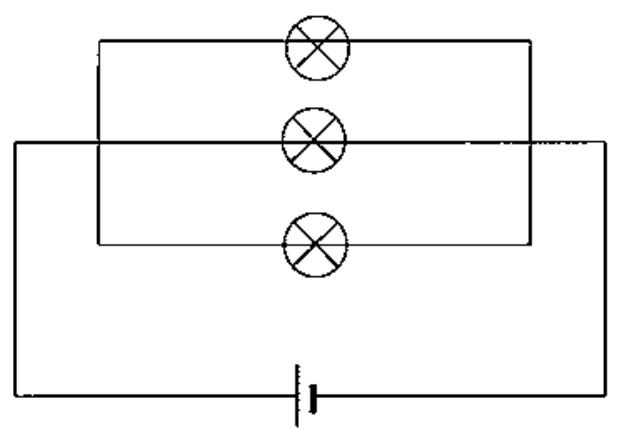

poco claros que habían proporcionado los estudios sobre coherencia y consistencia de las ideas de los alumnosun nivel de análisis donde la coherencia se da con claridad: el del compromiso ontológico con la causalidad (en el mundo ocurren cosas porque hay causas y efectos). El alumno razona en base a la existencia de causas y efectos que son los que dan cuenta de los cambios que se dan en en el mundo real. No necesita un compromiso epistemológico como el que pretendemos, como profesores, que asuman.

Estos resultados abren una puerta a la reinterpretación de los estudios de coherencia y consistencia de las ideas alternativas.

Por otra parte, con la técnica «teachback» aparece una nueva visión de las ideas científicas de los alumnos: una imagen mucho más dinámica (Fig. 3) que la que se desprende de las respuestas a los cuestionarios convencionales.

En definitiva, a través de un adecuado diálogo puede llegarse a conocer suficientemente el modelo mental utilizado por un alumno y los motivos de su evolución. Estrategias adecuadas pueden facilitar tal evolución.

\section{Implicaciones didácticas: modificar el modelo mental}

Aunque todavía de forma incipiente, los modelos mentales causales parecen no sólo dar cuenta de los descubrimientos ya realizados en este terreno, sino que también parecen prometedores para:

- dar respuesta a algunos de los interrogantes actualmente planteados sobre la persistencia de las concepciones alternativas;

- dar un nuevo enfoque a las clases de ciencias.
Si puede generalizarse que los alumnos al enfrentarse a explicaciones cualitativas de ciencias se sirven de procesos causales y no legales, Ia utilización en clase de ciencias de un vocabulario próximo a la causalidad podría mejorar su comprensión. Sería más fácil conectar con los modelos mentales de los alumnos y hacernos, como profesores, más inteligibles.

Todo ello debería no comportar un afianzamiento de sus razonamientos lineales causales, como señala Rozier, sino que debería introducirse como etapa intermedia que permitiera conectar a los alumnos con lo que pretendemos enseñar. La ciencia no está asentada sobre explicaciones causales sino legales, pero hay que proceder a una «mediación» entre el pensamiento del alumno y la ciencia.

Está suficientemente establecido en la actualidad que conviene utilizar «conceptos mediadores» para hacer pasar de unas concepciones alternativas a concepciones científicas. Dicho de otro modo, el proceso de modelización (Joshua y Dupin, 1993) requiere establecer diversas etapas, dadas las progresiones y regresiones que se operan.

Nuestra meta será la de ir haciendo evolucionar los modelos mentales causales creando situaciones de clase en las que los alumnos tengan que enfrentarse con sus personales exigencias de coherencia, correspondencia y robustez. Si en el modelo mental que el alumno ha elaborado observa contradicciones, si con tal modelo no puede explicar satisfactoriamente cierta situación o considera que no puede aplicarlo a otras realidades, lo modificará para hacerlo más adecuado. Es así cómo evolucionan los modelos mentales y se acercan a los modelos de la ciencia.

Se intuye que en los próximos años se acudirá a modelos teóricos, como el anterior, para interpretar las concepciones y razonamientos de los alumnos y para abordar con unos fundamentos más sólidos la didáctica de las ciencias. 


\section{CONCLUSIÓN}

Las investigaciones sobre las concepciones de los alumnos pueden realizarse bajo distintos enfoques cada uno de los cuales conduce a planteamientos didácticos distintos. Los tres enfoques analizados en este artículo se resumen en el cuadro 1 .

Cuadro I

Características de los diferentes programas de investigación en didáctica de las ciencias.

\begin{tabular}{|c|c|c|c|}
\hline & CONCEPCIONES ALTERNATIVAS & FORMAS DE RAZONAMIENTO & MODELOS MENTALES \\
\hline $\begin{array}{l}\text { Inicio } \\
\text { aproximado }\end{array}$ & Finales de los setenta & Mediados de los ochenta & Inicio de los noventa \\
\hline $\begin{array}{l}\text { Técnica de } \\
\text { recogida } \\
\text { de datos }\end{array}$ & $\begin{array}{l}\text { Cuestionarios o entrevistas con } \\
\text { preguntas abjertas o cerradas }\end{array}$ & $\begin{array}{l}\text { Cuestionarios o entrevistas con } \\
\text { preguntas abiertas o cerradas }\end{array}$ & $\begin{array}{l}\text { Conversación hasta llegar a un } \\
\text { consenso sobre el pensamiento } \\
\text { del alumno "teachback" }\end{array}$ \\
\hline $\begin{array}{l}\text { Las } \\
\text { investigaciones } \\
\text { se centran en }\end{array}$ & $\begin{array}{l}\text { Las concepciones de los alumnos } \\
\text { (modos de ver) }\end{array}$ & $\begin{array}{l}\text { Los razonamientos lógico-causales } \\
\text { de los alumnos } \\
\text { (modos de razonar) }\end{array}$ & $\begin{array}{l}\text { Las representaciones causales } \\
\text { de los alumnos (modelos } \\
\text { mentales causales) }\end{array}$ \\
\hline $\begin{array}{l}\text { Se llega a los } \\
\text { tesultados } \\
\text { básicamente } \\
\text { medianfe un } \\
\text { proceso de }\end{array}$ & $\begin{array}{l}\text { Inducción a partir de los datos } \\
\text { (gencralizaciones empíricas, } \\
\text { leyes de bajo nivel) }\end{array}$ & $\begin{array}{l}\text { Inducción a partir de los datos } \\
\text { y de las concepciones de la } \\
\text { (leyes de alto nivel) }\end{array}$ & $\begin{array}{l}\text { Teorización en el campo de la } \\
\text { inteligencia artificial (teoría) }\end{array}$ \\
\hline Se descubren & $\begin{array}{l}\text { Gran variedad de concepciones } \\
\text { para dar cuenta de los datos }\end{array}$ & $\begin{array}{l}\text { Algunos patrones de razonamiento } \\
\text { para dar cuenta de las concepciones } \\
\text { y de los datos }\end{array}$ & $\begin{array}{l}\text { Un solo modelo teórico que } \\
\text { proporciona categorias } \\
\text { para describir los modelos } \\
\text { mentales de los alumnos. } \\
\text { Un modelo mental da cuenta } \\
\text { de las formas de razonamiento, } \\
\text { de las concepciones y de } \\
\text { los datos a los que va asociado }\end{array}$ \\
\hline $\begin{array}{l}\text { Aprender } \\
\text { ciencias será, } \\
\text { principalmente }\end{array}$ & $\begin{array}{l}\text { Cambiar las concepciones } \\
\text { por otras más cientificas } \\
\text { (cambio conceptual) }\end{array}$ & $\begin{array}{l}\text { Cambiar los tazonamientos y las } \\
\text { concepciones por otros aceptados } \\
\text { científicamente } \\
\text { (noción de objeto-obstáculo) }\end{array}$ & $\begin{array}{l}\text { Introducir modificaciones en el } \\
\text { modelo mental para mejorarlo } \\
\text { garantizando el cumplimiento } \\
\text { del principio causal }\end{array}$ \\
\hline $\begin{array}{l}\text { Provocan el } \\
\text { cambio de } \\
\text { concepcion, de } \\
\text { razonamiento o } \\
\text { de modelo }\end{array}$ & $\begin{array}{l}\text { El deficiente estatus de las } \\
\text { concepciones (referente a } \\
\text { insatisfaccion, inteligibilidad, } \\
\text { plausibilidad y aplicabilidad) }\end{array}$ & $\begin{array}{l}\text { La insuficiencia de los } \\
\text { razonamientos requeridos para } \\
\text { analizar situaciones más complejas }\end{array}$ & $\begin{array}{l}\text { La falta de coherencia, } \\
\text { correspondencia o robustez } \\
\text { de modelo mental } \\
\text { inicialmente empleado }\end{array}$ \\
\hline
\end{tabular}

\section{REFERENCIAS BIBLIOGRÁFICAS}

ALIBERAS, J., GUTIÉRREZ, R. e IZQUIERDO, M. (1989). La didàctica de les ciències: una empresa racional. Enseñanza de las Ciencias, 7(3), pp. 277-284.

ASTOLFJ, J.P. (1993). Los obstáculos para el aprendizaje de conceptos en ciencias: la forma de franquearlos didácticamente.
Diez años de investigación e innovación en enseñanza de las ciencias, CIDE 1983-93. Col. Investigación, núm. 86, MEC.

BACHELARD, G. (1938). La formation de l'esprit scientifique. París:Vrin. 
CARAMAZZA, A., McCLOSKEY, M. y GREEN, B. (1981). Naive beliefs in sophisticated subjects: misconceptions about trajectories of objects, Cognition, 9, pp. 117-123

CLEMENT, I. (1983). Students' alternative conceptions in Mechanics: A coherent system of preconceptions? Proceedings of "Misconceptions in Science and Mathematics. Nueva York: Cornell University, Ithaca.

CLOSSET, J.L. (1983). Le raisonnement séquentiel en elèctrocinetique. $\mathrm{PhD}$. Université Paris VII

DE KLEER, J. y BROWN, J.S. (1981). Mental models of physical mechanism and their acquisition, en Anderson, J.R. (ed.), Cognitive skills and their acquisition, pp. 285-309. Hillsdale, N.J.: LEA.

DE KLEER, I. y BROWN, J.S. (1983). Assumptions and ambiguities in mechanistic mental models, en Gentner,D. y Stevens,A.L. (eds.), Mental models, pp. 155-190. Hillsdale, N.I.: LEA.

DRIVER, R. (1981). Pupils' alternative frameworks in science. European Journal of Science Education, 3(1), pp. 93-101.

DRIVER, R. (1981).The pupil as Scientists? The Open University Press. Milton Keynes, U.K.

DRIVER, R. (1985). Cognitive psychology and pupils' frameworks in mechanics, en Lijnse (ed.), The Many Faces of Teaching and Learning Mechanics. Utrech: GIREP/SVO/UNESCO.

DRIVER, R. (1986). Psicologíacognitiva y esquemas conceptuales de los alumnos. Enseñanza de las Ciencias, 4(1), pp. 3-15.

DRIVER, R. y EASLEY, J. (1978). Pupils and paradigms: a review of literature related to concept development in adolescent science students. Studies in Science Education, 5, pp. 61-84.

DRIVER, R. y ERICKSON, G. (1983). Theories-in-action: some theoretical and empirical issues in the study of students' conceptual frameworks in science. Studies in Science Education, 10, pp. $37-60$.

DRIVER, R., GUESNE, E. y TIBERGHIEN, A. (1989). ldeas científicas en la infancia y la adolescencia. Madrid; MECMorata.

DUIT, R. (1990). Students' conceptual frameworks. Consequences for learning science, en Glynn, S., Yeany, R., Britton, B. (eds.), The psycology of learning science, Hillsdale: Erlbaum.

DUIT, R. (1993). Researchon students' conceptions, developments \& trends. Paper presented at the Third International Seminar on Misconceptions and Educational Strategies in Science and Mathematics. Cornell University, Ithaca, EEUU.

DUPIN, J.J. y JOSHUA, S. (1987). Conceptions of French pupils concerning electric circuits: structure and evolution. J. Res. Sci. Teach., 24(9), pp. 791-806.

EQUIP ICE (1993). Ciencies experimentals. Del disseny curriculara l'aula. ICE Universitat Autonoma de Barcelona.

ENGEL, E. y DRIVER, R. (1986). A study of consistency in the use of students' conceptual frameworks across different tasks contexts. Science Education, 70(4), pp. 473-496.

FAUÇONNET, S. (1984). Étude de résolution de problèmes analogues, en Delacote, G. et al. (eds.), Research on Physics Education. París: (CNRS): La Londe les Maures.
FINEGOLD, M. y GORSKY, P. (199I). Students' concepts of force as applied to related physical systems: a search for consistency. Int. J. Sci. Educ., 13(1), pp. 97-113.

GENTNER, D. y STEVENS, A.L. (eds.)(1983). Mental models. Hillsdale, N.J.: LEA.

GIL, D. (1986). La metodología científica y la enseñanza de les ciencias. Unas relaciones controvertidas. Enseñanza de las Ciencias, 4(2), pp. 111-121.

GIL, D. (1994). Diez años de investigación en didáctica de las ciencias: realizaciones y perspectivas. Enseñanza de las Ciencias, I2(2), pp. 154-164.

GUIÉRREZ, R. (1994). Coherencia del pensamiento espontáneo y causalidad. El caso de la dinámica elemental. Tesis doctoral. Madrid: Universidad Complutense.

GUTIÉRREZ, R. y OGBORN, J. (1992). A causal framework for analysing alternative conceptions. Int. J. Scie. Educ., $14(2)$, pp. 201-220.

HASHWEH, M.Z. (1986). Toward an explanation of conceptual change. European Journal of Science Education, 8(3), pp. 229-249.

HEWSON, P.W. (1985). Epistemological commitments in the learning of science: examples from dynamics. European Journal of Science Education, 7(2), pp. I63-172.

HEWSON, M.G. y HAMLYN, D. (1984). The influence of intellectual environment on conceptions of head. Eur. J. Sci. Educ., 6(3), pp. 245-262.

HEWSON, P.W. y THORLEY, N.R. (1989). The conditions of conceptual change. International Joumal of Science Education, 11, pp. 541-553.

HIERREZUELO, J. y MONTERO, A. (1988). La ciencia de los alumnos. Barcelona: Laia / Cuadernos de Pedagogía.

INHELDER, B. y PIAGET, J. ( I955). De la logique de l'enfant à la logique de l'adolescent. París: PUF.

JOHNSON-LAIRD, P.N. (1983). Mental models. Cambridge: Cambridge University Press.

JOHNSON-LAIRD, P.N. (1985). Mental models, en Aitkenhead, A.M. y Slack, J.M. (eds.), Issues in cognitive modelling, pp. 8I-99. Londres: LEA. Open University Press.

JOSHUA, S. y DUPIN, J.J. (1993). Introduction à la didactique des sciences et des mathematiques. París: PUF.

LICHT, P. y THIJS, G.D. (1990). Method to trace coherence and persistence of preconceptions. Int. J. Sci. Educ., 12(4), pp. $403-416$.

MALI, G.B. y HOWE, A. (1979). Development of Eaxth and Gravity concepts among Nepali children. Science Education, 63(5), pp. 685-691.

MARTINAND, J.L. (1986), Connaître et transformer la matière. Berna: Peter Lang.

MAURINES, L. (1986). Spontaneous reasoning on the propagation of visible mechanical signals. Int. J. Sci. Educ., 14(3), pp. $279-293$.

MILLAR, R. (1994). Alternative frameworks or context-specific reasoning? School Science Review, 75 (272), pp. 27-34. 
NORMAN, D. (1990). La psicología de los objetos cotidianos. Madric: Nerea.

NUSSBAUM, J. y NOVAK, J.D. (1976). Children's conception of the Earth as a cosmic body: a cross-age study. Science Education, 63(1), pp. 83-93.

PINTÓ, R. (1991). Algunos conceptos implícitos en la primera y segunda leyes de la termodinámica. Capítulo 7. Tesis doctoral. Universidad Autónoma de Barcelona

POSNER, G. etal. (1982). Accomodation of a scientific conception: toward a theory of conceptual change. Science Education, 66(2), pp. 211-227.

POZO, J. I. (1992). Las ideas de los alumnos sobre la ciencia como teorias implícitas. Infancia y Aprendizaje, 57, pp. $3-22$.

REIF, F. y LARKIN, J.H. (I991). Cognition in scientific and everyday domains: comparison and learning implications. Journcl of Research in Science Education, 28(9), pp. 733-760.

RESNICK, L.B. (I989). Knowing Learning and Instruction. Hillsdale, N.J.: LEA.

ROGERS, Y. (1992). Mental models and complex tasks, en Rogers, Y., Rutherford, A. y Bibby, P.A. (eds.), Models in the mind, pp. 1-7, 145-149. Londres: Academic Press.
ROZIER, S. (1988). Le raisonnement linéaire causal en thermodynamique élémentaire. PhD., Université Paris VII.

SANMARTÍ, N. (1989). Dificultats en la comprensió de la diferenciaci6 entre els conceptes de mescla i compost. Tesis doctoral. Universidad Autónoma de Barcelona.

SHIPSTONE, D.M. et al. (1988). A study of students' understanding of electricity in five European countries. Int. J. Sci. Educ., 10(3), pp. 303-316.

THAGARD, P. (1988). Computational philosophy of science. Cambridge: Bradford.

TOULMIN, S. (1977). La comprensión humana (I). Madrid: Alianza.

VIENNOT, L. (1988). Tendance à la réduction functionelle: obstacle au savoir scientifique et object de consensus, en Construction des savoirs, obstacles et conflits, pp. 84-91. Montréal: CIRADE, Agence d'Arc inc.

VIENNOT, L. (1977). Le raisonnement spontané en dynamiquue élémentaire. Tesis doctoral. Université Paris VII.

WEST, L.H.T. y PINES, A.L. (1983). How «rational» is rationality? Science Education, 67(1), pp. 37-39.

[Artículo recibido en junio de 1995 y aceptado en abril de 1996.] 